\title{
STRICT LIABILITY IN A PRINCIPAL-AGENT MODEL
}

\author{
HARRY A. NEWMAN \\ Assistant Professor, University of Illinois at Chicago, College of Business \\ Administration, Chicago, IL 60680
}

AND

DAVID W. WRIGHT

Assistant Professor, The University of Michigan, School of Business Administration, Ann Arbor, MI 48109

\section{INTRODUCTION}

Previous analytical work examining strict liability has generally ignored the existence of moral hazard problems within the firm. In this work, the firm has been implicitly modeled as a single individual (for example, Shavell [1980, 1982], Landes and Posner [1981], Polinsky [1980], Polinsky and Rogerson [1983], and Mantell [1984]). ${ }^{1}$ However, it is often the case that an owner of a firm (principal) hires an employee (agent) to perform activities which could result in the harm of a third party. For example, in the recent industrial accident case of the 1984 Bhopal, India gas leak, Union Carbide Corporation had established the subsidiary Union Carbide India Ltd., which in turn hired Indian employees to operate a chemical plant. In general, if the preferences of the agent differ from those of the owner and if the agent's effort is unobservable, then a moral hazard problem may exist within the firm. This study examines the socially optimal level of care and the care taken under strict liability in the presence of moral hazard through the application of principal-agent economic models. ${ }^{2}$

Since this paper examines environments in which a principal hires an agent to engage in a productive activity, the influence that strict liability has on firm behavior can be analyzed in settings where firm ownership is separated from management (for example, as in the Union Carbide Bhopal, India accident). While previous research has considered the direct influence of liability rules on the care taken by an owner-manager, this paper examines the influence that strict liability

We are grateful for the insightful comments of Greg Niehaus, Jim Noel, Ted Snyder, and Robert Thomas.

'In the prior literature, the firm could be viewed as a collection of individuals whose preferences are identical. Analytically, there is no substantive difference between a firm comprised of a single individual and a firm consisting of a collection of individuals with identical preferences. The economics literature has long recognized that preferences may, in fact, differ across individuals. This paper demonstrates that moral hazard arising from a difference in preferences and effort unobservability can affect the level of care taken by the firm.

${ }^{2}$ Essentially, under a strict liability rule, a party which causes a loss for a victim must pay damages whether or not the party was negligent.

(C) 1990 Butterworth-Heinemann 
has on the choice of employment contracts offered by the owner to the agent, which in turn, influences the agent's choice of care. ${ }^{3}$

This paper finds that strict liability induces the owner to offer employment contracts that motivate the agent to take a socially optimal level of care. It is also found that the care exerted by the agent when moral hazard is present can be less than or greater than the care taken when moral hazard is absent.

The remainder of this paper is organized as follows. Section 2 presents the fundamental principal-agent model employed in the paper. Section 3 considers the social welfare maximization problem. Section 4 uses the model developed in section 2 to compare the level of care taken by the agent under strict liability with the social welfare maximizing level of care. Section 5 compares the effort exerted when moral hazard is absent with the effort exerted when moral hazard is present under strict liability. The section also examines how the presence of moral hazard affects the socially optimal level of care. Conclusions and implications are given in section 6.

\section{THE PRINCIPAL-AGENT MODEL OF A FIRM ENGAGED IN HAZARDOUS ACTIVITIES}

Suppose a risk neutral principal hires a risk averse agent for his productive effort. Both the principal and agent act to maximize their respective individual expected utilities. The agent manages a potentially hazardous endeavor (for example, blasting activities or the operation of a chemical plant) where there is a probability that such activities can result in off-site property damage. ${ }^{4}$ The level of effort chosen by the agent affects the likelihood of an accident. Thus, effort can be interpreted as care. It is also assumed that it is too costly for a potential victim to unilaterally avoid damages caused by an accident. Thus, the socially optimal solution will involve the exercise of care by the firm in the prevention of an accident. The following notation is used in the paper.

$$
\begin{aligned}
a= & \text { the level of care taken by the agent in managing the hazardous } \\
& \text { activity. } \\
p(a)= & \text { the probability of an accident given the agent's level of care. } \\
W(I)= & \text { the agent's compensation as a function of } I \text {, where } I \text { is some } \\
& \text { mutually obscrvable variable upon which an enforceable contract } \\
& \text { can be based. } \\
H(W(I), a)= & \text { the agent's utility function as a function of compensation and } \\
& \text { effort. } \\
u= & \text { the agent's reservation level of utility, where } u>0 . \\
R= & \text { the firm's (principal's) net profits prior to compensation payments } \\
& \text { to the agent and any legal damages that might be payable to the } \\
& \text { victim if an accident occurs. }
\end{aligned}
$$

\footnotetext{
${ }^{3}$ Principal-agent models have been used to examine the economic effects of the imposition of a vicarious liability rule on firm behavior (Sykes 1981, 1984; Kornhauser, 1982). Throughout this paper a vicarious liability rule is employed since it is the extant rule of law. Thus, principals are assumed to be held liable for the torts of their agents.

${ }^{4}$ Damages for personal harm can also be incorporated into the model if there is a monetary equivalent for personal harm incurred as a result of an accident.

${ }^{5}$ The results of the paper would not change if $R$ is made dependent on whether an accident occurs (i.e., the firm might incur costs resulting from an accident due to the loss of assets and impairment of operations).
} 
$G(\cdot)=$ the potential victim's utility function where the argument is the level of total wealth.

$y=$ the potential victim's initial wealth endowment (that is, wealth prior to any accident).

$L=$ the monetary loss sustained by a victim if an accident occurs.

The following assumptions will be employed in the paper.

(A1) The agent's feasible action set: The agent chooses an action from a set of actions represented by a closed real interval, $[a, \bar{a}]$.

(A2) The accident prevention technology: Regardless of the level of care taken by the agent, an accident is never certain nor impossible. Furthermore, the probability of an accident is strictly decreasing in care, but at a decreasing rate. Thus, $0<p(a)<1, p^{\prime}(a)<0$, and $p^{\prime \prime}(a)>0$ for all $a \in\lfloor a, \bar{a}]$ where $p^{\prime}(a)$ and $p^{\prime \prime}(a)$ are the first and second derivatives of $p(a)$, respectively. This assumption is consistent with the prior literature (for example, Shavell [1980, 1982, 1984]).

(A3) The agent's utility: The agent is risk and effort averse and has a utility function which is separable in wealth and effort. Thus, $H(W(I), a)=$ $U(W(I))-a$, with $U^{\prime}(\cdot)>0$ and $U^{\prime \prime}(\cdot)<0$ where $U^{\prime}(\cdot)$ and $U^{\prime \prime}(\cdot)$ are the first and second derivatives of $U(\cdot)$, respectively. ${ }^{6}$ Let $U^{-1}$ represent the inverse of $U(\cdot)$ with $U^{-1 \prime}(\cdot)$ and $U^{-1 \prime \prime}(\cdot)$ denoting the first and second derivatives of $U^{-1}(\cdot)$, respectively. ${ }^{7}$

(A4) The principal's utility: The principal is risk neutral.

(A5) The victim's utility: The victim is risk averse so that $G^{\prime}(\cdot)>0$ and $G^{\prime \prime}(\cdot)<$ 0 where $G^{\prime}(\cdot)$ and $G^{\prime \prime}(\cdot)$ are the first and second derivatives of $G(\cdot)$, respectively.

(A6) Moral hazard: The agent's efforts cannot be observed.

(A6') No Moral hazard: The agent's efforts are observable.

In order to introduce moral hazard into the principal-agent model, it will generally be assumed that the agent's efforts cannot be observed (A6). Thus, the employment contract offered to the agent must be based on some other variable which is observable. Since an accident is observable and its likelihood is influenced by the agent's effort, the occurrence of an accident itself can serve as a variable upon which an enforceable and motivational contract can be based. Thus, let $I=1$ if an accident occurs and $I=0$ otherwise.

The analysis of section 5 will at times utilize assumption $\left(\mathrm{A6}^{\prime}\right)$ in order to examine the impact moral hazard has on optimal employment contracts and levels of care compared to what occurs when moral hazard is absent.

The principal's task is to offer an incentive compatible contract to the agent which maximizes the principal's expected utility given the firm operates under a strict liability system. Solutions to the problem are considered in section 4 .

\section{THE SOCIAL WELFARE MAXIMIZATION PROBLEM}

The social welfare maximization problem is provided so that the social welfare maximizing level of care can be compared with the care taken under strict liability. A social planner would maximize a weighted average of the victim's and the

${ }^{6}$ The assumption that the agent's utility is linear in effort is made without loss of generality since units of effort can always be chosen so that this is true.

${ }^{7}$ Since $U^{\prime}>0$ and $U^{\prime \prime}<0, U^{-1}$ exists and is strictly increasing and convex. 
principal's utility functions. ${ }^{8}$ This is equivalent to maximizing one party's expected utility subject to the other earning his reservation level of utility as in Shavell [1982]. If the agent's level of care is unobservable, the tools available to the social planner for this task consist of the choice of social welfare maximizing employment contracts and the direct transfer of wealth between the principal and victim, contingent upon the occurrence of an accident. Let $t_{1}\left(t_{2}\right)$ represent the wealth transfer between the principal and potential victim if an accident occurs (does not occur). Let $c$ and $1-c$ be the weights applied to the principal's utility function and the agent's utility function, respectively, where $0<c<1$.

The socially optimal level of care is found by solving the following maximization problem:

$$
\begin{aligned}
& \max _{W(I), a, t_{1}, t_{2}} c\left[R-E(W(I))-p(a) t_{1}-(1-p(a)) t_{2}\right] \\
&+(1-c)\left[p(a) G\left(y+t_{1}-L\right)+(1-p(a)) G\left(y+t_{2}\right)\right]
\end{aligned}
$$

Subject to

$$
\begin{aligned}
& E[U(W(I))]-a \geq u \\
& a \in \operatorname{argmax} E[U(W(I))]-\dot{a} \\
& a \mathfrak{a} \in[\underline{a}, \bar{a}]
\end{aligned}
$$

where $E$ is the expectation operator.

It is useful to note several points about this social welfare problem and its solution. First, the objective function is linearly decreasing in the expected compensation of the agent, $E(W(I))$. Second, the first order conditions, with respect to $t_{1}$ and $t_{2}$, imply $t_{2}=t_{1}-L$. Consequently, the potential victim's wealth will be $y+t_{2}=y+t_{1}-L$ whether an accident occurs or not, a well-known result of optimal risk sharing imposing no risk on the risk averse potential victim. Therefore, the objective function can be rewritten as

$$
c\left[R-E(W(I))-p(a) L-t_{2}\right]+(1-c) G\left(y+t_{2}\right) .
$$

Third, $t_{2}$ solves the expression $G^{\prime}\left(y+t_{2}\right)=c /(1-c)$ and hence does not depend on the contract, $W(I)$, or the agent's level of care, $a$. If the social planner does not desire to transfer wealth in the absence of an accident, $c$ can be chosen so that the optimal value for $t_{2}$ is 0 . Finally, the assumptions that (1) the probability of an accident decreases as more care is taken $\left(p^{\prime}<0\right)$, (2) the marginal benefits from additional care are decreasing $\left(p^{\prime \prime}>0\right)$, and (3) the agent is risk averse (also employed by Shavell $[1980,1982,1984])$ meet the sufficient conditions given in Rogerson [1985] to guarantee that the agent's effort choice is unique (that is, given an employment contract, the set of actions maximizing the agent's expected utility consists of a single element).

Intuitively, the optimal employment contract equates society's expected marginal bencfits from additional care with society's expected marginal costs. The expected marginal benefits consist of the change in the probability of an accident multiplied by $L$. The expected marginal costs consist of the additional expected

${ }^{8}$ In fact, the social welfare between the principal, agent and potential victim is being managed. The welfare of the agent is governed by $u$ and can be interpreted as a variable under the control of the social planner. 
compensation necessary to induce the agent to exert greater effort in the prevention of an accident.

\section{STRICT LIABILITY UNDER MORAL HAZARD}

This section compares the effort exerted under strict liability with the socially optimal level of care. Under strict liability the principal must pay damages, $L$, in the event of an accident regardless of the level of care taken by the agent. Thus, the principal's maximization problem can be stated as:

$$
\max _{W(I), a} R \quad E(W(I)) \quad p(a) L
$$

Subject to

$$
\begin{aligned}
& E[U(W(I))]-a \geq u \\
& a \in \operatorname{argmax} E[U(W(I))]-\dot{a} \\
& \quad a ́(\underline{a}, \bar{a}]
\end{aligned}
$$

Proposition 1 compares the effort exerted by the agent under strict liability with the socially optimal level of care.

Proposition 1 Given (A1)-(A5) and the existence of moral hazard (A6), a strict liability rule induces the principal to offer an employment contract which motivates the agent to provide a socially optimal level of care.

Proof: The constraints in the social welfare optimization problem (1) and in the principal's maximization problem under strict liability (2) are identical. Thus, solutions to one problem are feasible in the other. As noted earlier since $y+$ $t_{2}=y+t_{1}-L$, the social welfare objective function reduces to:

$$
\max _{a, W(I), t_{2}}\{c[R-E(W(I))-p(a) L]\}+\left\{(1-c) G\left(y+t_{2}\right)-c t_{2}\right\}
$$

Since $t_{2}$ was shown not to depend on $a$ or $W(I)$, the objective function is separable into two independent terms:

$$
\begin{gathered}
\max _{a, W(I)} c[R-E(W(I))-p(a) L] \\
\max _{t_{2}}(1-c) G\left(y+t_{2}\right)-c t_{2}
\end{gathered}
$$

The first of these two expressions is the principal's objective function multiplied by the weighting factor, $c$. Thus, optimizing contracts in the principal's problem (2) also serve to maximize the social welfare function in (1).

The following lemma characterizes the optimal employment contract under strict liability and will be useful in section 5 when examining the effects of moral hazard on strict liability models. Let an employment contract in the presence of moral hazard be of the form $W(I)=\{W(0), W(1)\}$ where $W(0)$ is the compensation paid to the agent if no accident occurs and $W(1)$ is the compensation in the event of an accident. 
Lemma 1 Given (AI)-(A4) and the existence of moral hazard (A6),

(I) The employment contract, $W_{a}(I)$, inducing the level of care, a, that minimizes expected labor costs, $E C(\hat{a})=[p(a) w(1)+(1-p(a)) w(0)]$ is:

$$
\begin{aligned}
& W_{\dot{a}}(I)=w(0)=w(1)=U^{-1}(u+\underline{a}) \\
& W_{\dot{a}}(I)= \begin{cases}w(0)=U^{-1}\left(u+\dot{a}-\frac{p(a ́)}{p^{\prime}(a)}\right) & \text { for } \dot{a}=\underline{a} \\
w(1)=U^{-1}\left(u+\dot{a}+\frac{1-p(a)}{p^{\prime}(a ́ a)}\right) & \text { for } \dot{a} \neq \underline{a}\end{cases}
\end{aligned}
$$

(2) EC(a) is strictly increasing in the induced level of care, a.

Proof: See Appendix 1.

\section{THE EFFECTS OF MORAL HAZARD ON STRICT LIABILITY MODELS}

The previous section examined the influence of strict liability on firm behavior in the presence of moral hazard. This section examines how the results in the presence of moral hazard compare with those when moral hazard is absent.

The first step is to derive principal-agent models of the accident problem where moral hazard is absent. This is accomplished by relaxing assumption A6 and assuming the agent's effort is observable $\left(\mathrm{A6}^{\prime}\right)$. It is then seen how the presence of moral hazard affects the socially optimal level of care and the care taken under strict liability.

An enforceable employment contract can be based on effort when it is observable. Denote a contract in which the agent's compensation is a function of (possibly) both the agent's action and the occurrence of an accident by $S(a, l)$.

The socially optimal level of care, when the agent's effort is observable, is found by solving the following maximization problem:

$$
\begin{aligned}
& \max _{S(a, I), a, f_{1},,_{2}} c\left[R-E(S(a, I))-p(a) t_{1}-(1-p(a)) t_{2}\right] \\
& +(1-c)\left[p(a) G\left(y+t_{1}-L\right)+(1-p(a)) G\left(y+t_{2}\right)\right]
\end{aligned}
$$

Subject to

$$
\begin{aligned}
& E[U(S(a, I))]-a \geq u \\
& a \in \operatorname{argmax} E[U(S(\dot{a}, I))]-a \\
& a \dot{a} \in[\underline{a}, \bar{a}]
\end{aligned}
$$

The principal's maximization problem under strict liability is given by:

$$
\max _{S(a . I) . a} R-E[S(a, I)]-p(a) L
$$


Subject to

$$
\begin{aligned}
& E[U(S(a, I))]-a \geq u \\
& a \in \operatorname{argmax} E[U(S(a,, I))]-a ́ \\
& \quad \dot{a} \in[\underline{a}, \bar{a}]
\end{aligned}
$$

The following lemma regarding the optimal employment contract between the principal and the agent is used in proving the main results of this section.

Lemma 2 Given $(A I)-(A 4)$ and the absence of moral hazard $\left(A 6^{\prime}\right)$ :

(1) The employment contract, $S_{\dot{a}}(a, I)$, inducing a given level of care, a, that minimizes expected labor costs is the forcing contract:

$$
S_{a}(a, I)= \begin{cases}s(a, I)=U^{-1}(u+a ́) & \text { if } a=a ́ a \\ 0 & \text { otherwise }\end{cases}
$$

(2) $s(a ́, I)=U^{-1}(u+a ́)$ is strictly increasing and convex in á.

Proof: See Appendix 1.

The following proposition is a restatement of Proposition 1 from section 4 but for firm environments in which moral hazard is absent. It is provided for completeness and for use in proving the main results of this section.

Proposition 2 Given (AI)-(A5) and the absence of moral hazard (A6'), a strict liability rule induces the principal to offer an employment contract which motivates the agent to provide a socially optimal level of care.

The proof for Proposition 2 is identical to the proof for Proposition 1, except for notation. It is omitted for purposes of brevity.

Proposition 3 states the main result of this section. Proposition 3 compares the socially optimal level of care in the presence of moral hazard with the socially optimal level of care in the absence of moral hazard. The proposition also compares the level of care induced by the principal under strict liability when moral hazard is present and absent. The proof of Proposition 3 assumes that the induced action under strict liability is interior to the feasible action set, so that the first derivatives of the objective functions equal zero. Let $a_{u}^{*}$ denote the level of care induced by the optimal employment contract in the solution to the principal's strict liability problem (2) when effort is unobservable. Let $a_{0}^{*}$ denote the level of care induced by the optimal employment contract in the solution to the principal's strict liability problem (4) when effort is observable.

Proposition 3 Given $(A l)-(A 5)$,

(I) Under strict liability, the care induced by the optimal employment contract offered in the presence of moral hazard, $a_{u}^{*}$, can be either greater than, equal to, or less than the care induced by the optimal employment contract in the absence of moral hazard, $a_{o}^{*}$.

(i) If $\frac{d}{d a} E C\left(a_{u}^{*}\right)>U^{-1^{\prime}}\left(u+a_{u}^{*}\right)$, then the induced level of care will be 
smaller in the presence of moral hazard.

(ii) If $\frac{d}{d a} E C\left(a_{u}^{*}\right)=U^{-1 \prime}\left(u+a_{u}^{*}\right)$, then the induced level of care will be the same in the presence and absence of moral hazard.

(iii) If $\frac{d}{d a} E C\left(a_{u}^{*}\right)<U^{-1 \prime}\left(u+a_{u}^{*}\right)$, then the induced level of care will be greater in the presence of moral hazard.

(2) The socially optimal level of care in the presence of moral hazard can be either greater than, equal to, or less than the socially optimal level of care in the absence of moral hazard.

(i) If $\frac{d}{d a} E C\left(a_{u}^{*}\right)>U^{-1 \prime}\left(u+a_{u}^{*}\right)$, then the socially optimal level of care will be smaller in the presence of moral hazard.

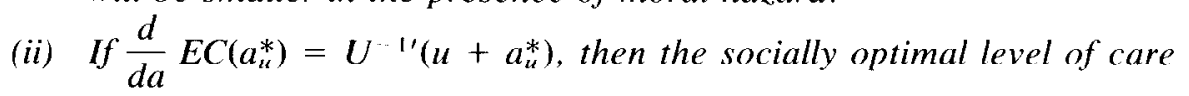
will be the same in the presence and absence of moral hazard.

(iii) If $\frac{d}{d a} E C\left(a_{u}^{*}\right)<U^{-1 \prime}\left(u+a_{u}^{*}\right)$, then the socially optimal level of care will be greater in the presence of moral hazard.

Proof:

(1) (i) Since $a_{u}^{*}$ and $a_{o}^{*}$ maximize the principal's expected utility for the two strict liability problems, they satisfy the first order conditions from the two maximization problems:

$$
\begin{array}{r}
-p^{\prime}\left(a_{u}^{*}\right) L-\frac{d}{d a} E C\left(a_{u}^{*}\right)=0 \\
-p^{\prime}\left(a_{o}^{*}\right) L-U^{-\prime^{\prime}}\left(u+a_{o}^{*}\right)=0
\end{array}
$$

However, if

$$
\frac{d}{d a} \mathrm{EC}\left(a_{u}^{*}\right)>U^{-1 \prime}\left(u+a_{u}^{*}\right)
$$

then

$$
-p^{\prime}\left(a_{u}^{*}\right) L-U^{-1 \prime}\left(u+a_{u}^{*}\right)>0 .
$$

This implies $a_{o}^{*}>a_{u}^{*}$ since the principal's expected utility in the absence of moral hazard is concave in $a$.

The proofs for parts (1) (ii) and (1) (iii) of the proposition follow in similar fashion and are omitted for the sake of brevity.

(2) The proof is immediate from Propositions 1, 2, and 3(1).

It is, of course, true that for any given level of care, $a$, the expected compensation costs in the presence of moral hazard, $E C(a)$, exceed the compensation costs in the absence of moral hazard, $U^{-1}(u+\hat{a})$. In fact, the excess of EC(a) over $U^{-1}(u+a)$ can be regarded as the costs of moral hazard. However, the fact that moral hazard is "costly" at any induced effort level does not imply that the 
equilibrium solution to the agency problem produces a lower level of care in the presence of moral hazard than when moral hazard is absent. Proposition 3 demonstrates that the level of effort exerted under strict liability in the presence of moral hazard can be either greater than, equal to, or less than the effort exerted in the absence of moral hazard.

The intuition behind this result is as follows. The equilibrium solutions to the two principal-agent problems equate the marginal benefits from effort with their marginal costs. The marginal benefit of a unit of additional effort on the part of the agent is represented by the reduced expected loss from an accident, $p^{\prime}(a) L$. For any given level of effort, $a$, these marginal benefits are the same in the presence or absence of moral hazard. However, the marginal costs of inducing additional effort differ, depending upon the presence or absence of moral hazard. If at $a_{u}^{*}$ the marginal costs of effort in the presence of moral hazard are increasing at a faster rate than in the absence of moral hazard $\left(\frac{d}{d a} E C\left(a_{u}^{*}\right)>U^{-1^{\prime}}\left(u+a_{u}^{*}\right)\right)$, then the costs of moral hazard are increasing in effort. In other words, the marginal costs of inducing an additional unit of effort would be less in the absence of moral hazard than in the presence of moral hazard. This implies that the induced effort in the presence of moral hazard is less than the induced effort in the absence of moral hazard, since the marginal benefits of additional effort are the same in both circumstances. Of course, when the marginal costs of moral hazard at $a_{u}^{*}$ are decreasing $\left(\frac{d}{d a} E C\left(a_{u}^{*}\right)<U^{-1 \prime}\left(u+a_{u}^{*}\right)\right)$, then the reverse is true. This implies $a_{o}^{*}<a_{u}^{*}$, since the loss in benefits by inducing lower effort is less than the savings in compensation. Further, since Propositions 1 and 2 demonstrate that the socially optimal level of care and the induced level of care under strict liability are the same (either in the presence or absence of moral hazard) it follows immediately that the socially optimal level of care can either increase or decrease with the introduction of moral hazard in the firm. Finally, since the case involving higher induced effort in the presence of moral hazard $\left(a_{o}^{*}<a_{u}^{*}\right)$ may be counterintuitive, an example is provided in Appendix 2.

\section{SUMMARY AND CONCLUSIONS}

This paper uses a principal-agent economic model of the firm to analytically examine issues surrounding the use of strict liability for the accident problem. This includes examining the consequences of the presence of moral hazard within the firm on the level of care induced by the principal under strict liability and on the socially optimal level of care.

The results show that strict liability motivates the principal to offer a contract which induces a socially optimal level of care. This is true either in the presence or absence of moral hazard within the firm. The analysis demonstrates that the care exerted under strict liability when moral hazard is present is generally different from the care taken when moral hazard is absent. The induced level of care can be either greater or lower in the presence of moral hazard than in its absencedepending on the marginal costs of moral hazard with respect to increases in effort. Similarly, the socially optimal level of care can be either higher or lower in the presence of moral hazard than when moral hazard is absent.

\section{APPENDIX 1}

This appendix provides proofs to Lemmas 1 and 2 .

\section{Proof of Lemma 1:}


The principal's employment contract inducing the level of care, $a$, that minimizes expected labor costs is derived by solving the following minimization problem:

$$
\min _{w(0), n(1)} p(a ́) w(1)+(1-p(a ́)) w(0)
$$

Subject to

$$
\begin{gathered}
p(a) U(w(1))+(1-p(a)) U(w(0))-a ́ a \\
p(a ́) U(w(1))+(1-p(a)) U(w(0))-a \\
\geq p(a) U(w(1))+(1-p(a)) U(w(0))-a \text { for all } a \in[\underline{a}, \bar{a}]
\end{gathered}
$$

(1) (i) Consider $a=a$. Since $p^{\prime}<0$ and $p^{\prime \prime}>0$, a necessary and sufficient condition for satisfying the second constraint (c2) is

$$
p^{\prime}(\underline{a})[U(w(1))-U(w(0))]-1 \leq 0 .
$$

Thus, (c3) can replace (c2) to produce an equivalent minimization problem. Now, at $w(0)=w(1)=U^{-1}(u+a)$ constraint (cl) is binding while (c3) is not. Therefore, the Kuhn-Tucker sufficient condition for minimization is that there exists an $s \geq 0$ such that:

$$
\begin{array}{r}
p(\underline{a}) \quad s p(\underline{a}) U^{\prime}(w(1))=0 \\
1-p(\underline{a})-s(1-p(\underline{a})) U^{\prime}(w(0))=0
\end{array}
$$

which is satisfied by $s=1 / U^{\prime}(w(1))=1 / U^{\prime}(w(0))$.

(ii) Consider $a \in(a, \bar{a})$. Since $p^{\prime}<0$ and $p^{\prime \prime}>0$, a necessary and sufficient condition for satisfying the second constraint (c2) is

$$
p^{\prime}(a)[U(w(1))-U(w(0))]-1=0 .
$$

Thus, (c4) can replace (c2) to produce an equivalent minimization problem. Now, at

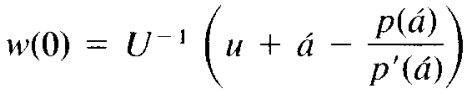

$$
\begin{aligned}
& w(1)=U^{-1}\left(\begin{array}{llll}
u & 1 & a & 1-p(a ́) \\
& & p^{\prime}(a ́)
\end{array}\right)
\end{aligned}
$$

constraint (c4) is satisfied and (c1) is binding. Therefore, the KuhnTucker sufficient conditions for minimization are that there exist an $s \geq 0$ and a $t$ such that:

$$
\begin{array}{r}
p(\hat{a})-s p(a) U^{\prime}(w(1))-t p^{\prime}(a) U^{\prime}(w(1))=0 \\
1-p(a)-s(1-p(a)) U^{\prime}(w(0))+t p^{\prime}(a) U^{\prime}(w(0))=0
\end{array}
$$

which are satisfied by: 


$$
\begin{aligned}
& s=\frac{(1-p(a ́)) U^{\prime}(w(1))+p(\hat{a}) U^{\prime}(w(0))}{U^{\prime}(w(0)) U^{\prime}(w(1))} \\
& t=-\frac{p(a))(1-p(a ́))\left[U^{\prime}(w(1))-U^{\prime}(w(0))\right]}{p^{\prime}(a) U^{\prime}(w(0)) U^{\prime}(w(1))}
\end{aligned}
$$

(iii) Consider $a=\bar{a}$. Since $p^{\prime}<0$ and $p^{\prime \prime}>0$, a necessary and sufficient condition for satisfying the second constraint (c2) is

$$
p^{\prime}(\bar{a})[U(w(1))-U(w(0))]-1 \geq 0 .
$$

Thus, (c5) can replace (c2) to produce an equivalent minimization problem. Now, at

$$
\begin{aligned}
& w(0)=U^{-1}\left(u+\bar{a}-\frac{p(\bar{a})}{p^{\prime}(\bar{a})}\right) \\
& w(1)=U^{-1}\left(u+\bar{a}+\frac{1-p(\bar{a})}{p^{\prime}(\bar{a})}\right)
\end{aligned}
$$

both constraints (c1) and (c5) are binding. Therefore, the Kuhn-Tucker sufficient conditions for minimization are that there exist $s, t \geq 0$ such that:

$$
\begin{array}{r}
p(\bar{a})-s p(\bar{a}) U^{\prime}(w(1))-t p^{\prime}(\bar{a}) U^{\prime}(w(1))=0 \\
1-p(\bar{a})-s(1-p(\bar{a})) U^{\prime}(w(0))+t p^{\prime}(\bar{a}) U^{\prime}(w(0))=0
\end{array}
$$

which are satisfied by:

$$
\begin{aligned}
& s=\frac{(1-p(\bar{a})) U^{\prime}(w(1))+p(\bar{a}) U^{\prime}(w(0))}{U^{\prime}(w(0)) U^{\prime}(w(1))} \\
& t=-\frac{p(\bar{a})(1-p(\bar{a}))\left[U^{\prime}(w(1))-U^{\prime}(w(0))\right]}{p^{\prime}(\bar{a}) U^{\prime}(w(0)) U^{\prime}(w(1))}
\end{aligned}
$$

(2) The first derivative of expected labor costs with respect to á is:

$$
\begin{aligned}
& \frac{d}{d \dot{a}}\left\{p(\hat{a}) U^{-1}\left(u+\dot{a}+\frac{1-p(a)}{p^{\prime}(a ́)}\right)+(1-p(a ́)) U^{-1}\left(u+\dot{a}-\frac{p(a ́)}{p^{\prime}(a ́)}\right)\right\} \\
& =p^{\prime}(\dot{a})\left[U^{-1}\left(u+\dot{a}+\frac{1-p(\hat{a})}{p^{\prime}(\hat{a})}\right)-U^{-1}\left(u+\dot{a}-\frac{p(\hat{a})}{p^{\prime}(\hat{a})}\right)\right] \\
& +\frac{p(\hat{a}) p^{\prime \prime}(\hat{a})(p(a)-1)}{\left(p^{\prime}(\hat{a})\right)^{2}}\left[U^{-1}\left(u+a+\frac{1-p(a)}{p^{\prime}(\hat{a})}\right)\right. \\
& \left.-U^{-1}\left(u+\dot{a}-\frac{p(a)}{p^{\prime}(a)}\right)\right]
\end{aligned}
$$

which is strictly greater than zero by $0<p(a)<1, p^{\prime}<0, p^{\prime \prime}>0$ and $U^{-1}$ 
strictly increasing and convex in $a$.

\section{Proof of Lemma 2:}

(1) The employment contract, $S_{\dot{a}}(a, I)$, inducing the level of care, a, that minimizes expected labor costs is derived by solving the following minimization problem:

$$
\min _{s(a, 0), s(a, a, 1)} p(a ́) s(a, 1)+(1-p(a ́)) s(a ́, 0)
$$

Subject to

$$
\begin{aligned}
& p(a ́) U(s(\dot{a}, 1))+(1-p(a ́)) U(s(a ́, 0))-\dot{a}>u
\end{aligned}
$$

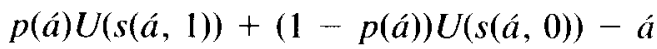

$$
\begin{aligned}
& \geq p(a) U(s(a, 1))+(1-p(a)) U(s(a, 0))-a \text { for all } a \in[\underline{a}, \bar{a}]
\end{aligned}
$$

From Harris and Raviv [1979], any contract based on $a$ and $I$ is equivalent to a forcing contract of the form:

$$
S_{\hat{a}}(a, I)= \begin{cases}s(a, I)=t(I) & \text { if } a=a ́ a \\ 0 & \text { otherwise }\end{cases}
$$

which induces the agent to take action $a$ such that

$$
p(a) U(t(1))+(1-p(a ́)) U(t(0))-a ́ a .
$$

Therefore, the contract minimizing expected labor costs is $t(1)=t(0)=$ $U^{-1}(u+a)$ since $U(\cdot)$ is concave. Hence, the agent's actual and expected compensation will be $U^{-1}(u+a)$.

(2) Since $U$ is strictly increasing, $U^{-1}$ is as well. Therefore, the first derivative of $s(\dot{a}, I)=U^{1}(u+\dot{a})$ with respect to $\dot{a}$ is positive.

\section{APPENDIX 2}

The purpose of this appendix is to provide an example that demonstrates that the level of care induced under strict liability can be larger when moral hazard is present than when moral hazard is absent.

Let $p(a)=(1-a)^{1.05}$ where $a \in[.01, .99], U(Z)=(1 / 10,000) Z^{8}$ where $Z$ is the agent's wealth, $u=.2, R=\$ 5,000,000, L=\$ 120,020$.

In this example, the optimal employment contract in the presence of moral hazard is $W(I)=\{\$ 124,974, \$ 7,555\}$ which induces the agent to exert effort $a_{u}^{*}=$ 9. When moral hazard is absent, the optimal contract pays the agent $\$ 80,537$ if effort level $a=.641$ is chosen and $\$ 0$ otherwise. The agent will then choose $a_{o}^{*}=.641$, of course. Therefore, in this example the effort induced in the presence of moral hazard is greater than in the absence of moral hazard.

\section{REFERENCES}

Brown, J. P., "Toward an Economic Theory of Liability," The Journal of Legal Studies, Vol. 2 (1973), pp. 323-49.

Harris, M. and $\Lambda$. Raviv, "Optimal Incentive Contracts with Imperfect Information," 
Journal of Economic Theory, Vol. 20 (1979), pp. 231-59.

Kornhauser, L. A., "An Economic Analysis of the Choice Between Enterprise and Personal Liability for Accidents," California Law Review, Vol. 70 (1982), pp. 1345-92.

Landes, W. M. and R. A. Posner, "The Positive Economic Theory of Tort Law," Georgia Law Review, Vol. 15 (1981), pp. 851-924.

Mantell, E. H., "Allocativc and Distributive Efficiency of Products Liability Law in a Monopolistic Market," Journal of Products Liability, Vol. 7 (1984), pp. 143-52.

Polinsky, A. M., "Strict Liability vs. Negligence in a Market Setting," The American Economic Review, Vol. 70 (1980), pp. 363-67.

Polinsky, A. M. and W. P. Rogerson, "Products Liability, Consumer Misperceptions, and Market Power," The Bell Journal of Economics, Vol. 14 (1983), pp. 581-89.

Rogerson, W. P., "The First-Order Approach to Principal-Agent Problems," Econometrica, Vol. 53 (1985), pp. 1357-67.

Shavell, S., "Risk Sharing and Incentives in the Principal and Agent Relationship," The Bell .Iournal of Economics, Vol. 10 (1979), pp. 55-73. $1-25$. "Strict Liability Versus Negligence," Journal of Legal Studies, Vol. 9 (1980), pp. $120-32$.

"A Model of the Optimal Use of Liability and Safety Regulation," Rand Journal of Economics, Vol. 15 (1984), pp. 271-80.

Sykes, A. O., "An Efficiency Analysis of Vicarious Liability Under the Law of Agency," The Yale Law Journal, Vol. 91 (1981), pp. 169-206. pp. 1231-80. 